\title{
Magnetic Monopoles in Hot QCD
}

\author{
C. P. Korthals Altes \\ Centre Physique Théorique au CNRS, \\ Luminy, 13288 Marseille Cedex, France \\ *E-mail: ab_altes@cpt.univ-mrs.fr
}

\begin{abstract}
In this talk we review how a dilute gas of magnetic monopoles in the adjoint describes the spatial $\mathrm{k}$-Wilson loops. We formulate an effective theory from $S_{M Q C D}$ by integrating out dof's down to scales in between the magnetic screening mass and the string tension and relate the $3 \mathrm{~d}$ pressure and the string tension. Lattice data are consistent with the gas being dilute for all temperatures.
\end{abstract}

Keywords: monopoles, QCD plasma, effective action.

\section{Motivation and outline}

The QCD plasma phase consists of colour electric quasi-particles, gluons, whose interactions get screened by the Debye effect. Their flux is not confined, like in a glueball.

The plasma is a nice theoretical laboratory to understand effects of quasi-particles on flux-loops. Flux-loops are either of the colour-electric variety (spatial 't Hooft loop) or of the magnetic variety (spatial Wilson loop).

Why do we mention "magnetic"? In QED plasmas there is no reason to suspect magnetic quasi-particles because long range static magnetic fields are possible, in contrast to the screening of long range electric fields. But in QCD there is indeed magnetic screening, as seen by lattice simulations. So there must be magnetic activity in the QCD plasma. That is corroborated by the behaviour of the spatial Wilson loop: through Stokes law it measures the flux of magnetic quasi-particles (MQP's or "monopoles")i Its thermal average behaves like an area law, as expected from a gas of free screened magnetic charges. In contrast to electric screening the magnetic screening is non-perturbative. The same is valid for the respective loops.

These magnetic charges are supposed to be in a representation of a 
global magnetic $\mathrm{SU}(\mathrm{N})$ group. They are non-perturbative objects. In order to produce a pressure proportional to $N^{2}-1$ (valid to all orders in perturbation theory) the monopoles be better in the adjoint representation. And this is enough to compute the tension for the Wilson loop in the totally antisymmetric representation built from $\mathrm{k}$ quark representations. Its highest weight $Y_{k}$ controls the Stokes form for the loop. The adjoint representation has a multiplicity $2 k(N-k)$ of non-zero charges $\pm 2 \pi / g$ in $\left.Y_{k}\right|_{a d j}$. Every magnetic charge contributes the same irrespective of the sign, and independently. Hence the tension is proportional to this multiplicity and this is up to 1 or 2 percent reproduced by the lattice data. In the last section we set up an effective theory for the monopole field.

\section{Dimensional reduction, and the magnetic sector}

Dimensional reduction is valid at temperatures well above $T_{c}$. We will be brief and only mention the essentials. Notation is $\mathrm{g}$ for the coupling, and $\mathrm{N}$ for the number of colours.Potentials are $\mathrm{NxN}$ matrices.

For the purpose of computing magnetic loops it reduction is particularly suitable. This is because the original 4d QCD action reduces at high $\mathrm{T}$ to 3d Magnetostatic QCD, the 3d Yang Mills theory:

$$
\begin{aligned}
S_{Q C D} & \rightarrow S_{E Q C D} \rightarrow S_{M Q C D}, \\
S_{E Q C D} & =\left(\vec{D} A_{0}\right)^{2}+m_{D}^{2} A_{0}^{2}+\lambda_{E} A_{0}^{4}+F_{i j}^{2}+\ldots \\
S_{M Q C D} & =F_{i j}^{2}+\ldots
\end{aligned}
$$

Both of the reduction steps are perturbative. The first integrates out the hard T modes, leaving only static modes. The resulting Electrostatic QCD Lagrangian is three dimensional and contains the $A_{0}$ potential as a massive excitation with the Debye mass $m_{D}^{2}=(N / 3) g^{2} T^{2}$, and of the $3 \mathrm{~d}$ Yang-Mills action, with the typical magnetic scale $g^{2} T$. The second step is valid when the Debye mass is much larger than the magnetic scale $g^{2} T$. Then we can integrate perturbatively the $A_{0}$ potential, and what stays is the 3d YangMills action $S_{M Q C D}$. This action has no perturbative components left at the scale $g^{2} T$.

\subsection{Magnetic observables}

These are the screening of the force between two Dirac $\mathrm{Z}(\mathrm{N})$ monopoles ${ }^{2}$ and the spatial Wilson loop. The first gives a temperature dependent screen- 
ing mass $m_{M}(T)$ :

$$
V_{M}(r)=d \frac{\exp \left(-m_{M} r\right)}{r} .
$$

It can be shown ${ }^{2}$ that this mass equals the mass gap in a $4 \mathrm{~d} \mathrm{SU}(\mathrm{N})$ gauge theory with one periodic space dimension of period $1 / T$. This relation is valid for all $T$, in particular $T=0$. At very large $T$ this mass can be simulated in $3 \mathrm{~d} S_{M Q C D}$, a considerable simplification.

The same magnetic activity that causes screening can be monitored by the spatial Wilson loop, that measures the average colour magnetic flux. The loop is carrying a representation $R$ built from a number $k$ of quark representations. Then the tension $\sigma_{k}$ is defined by:

$$
\left\langle W_{R}(C)\right\rangle=\int D A_{0} D \vec{A} \exp \left(i g \oint \vec{A}_{R} \cdot d \vec{l}\right) \sim \exp \left(-\sigma_{k} A\right)
$$

A the area of the minimal surface subtended by $\mathrm{C}$.

In three and four dimensions $\sigma_{k}$ only depends on the N-allity $k{ }^{\text {a }}$. So we need to discuss only one representation of $\mathrm{N}$-allity $k$, and we will limit ourselves to the fully antisymmetric one, built from $\mathrm{k}$ boxes. Its highest weight $Y_{k}$, written as a traceless $\mathrm{NxN}$ matrix, equals:

$$
Y_{k}=\frac{1}{N} \operatorname{diag}(k, k, \ldots, k, k-N, k-N, \ldots, k-N)
$$

This highest weight generalizes hypercharge. The average of the loop in this representation admits a Stokes law:

$\left\langle W_{k}(C)\right\rangle=\int D A_{0} D \vec{A} D \Omega \exp \left(i g \int d \vec{S} \cdot \operatorname{Tr}\left(\vec{B}-\frac{i}{g} \vec{D}(A) Y_{k}^{\Omega} \times \vec{D}(A) Y_{k}^{\Omega}\right) Y_{k}^{\Omega}\right)$.

The gauge transformation $\Omega$ acts on $Y_{k}$ as on a frozen Higgs field: $Y_{k}^{\Omega}=$ $\Omega Y_{k} \Omega^{\dagger}$. As such this integral will only converge when we admit a Higgs field that fluctuates around its frozen value ${ }^{3}$, i.e. a Higgs phase with the unbroken components $S U(k) \times S U(N-k) \times U(1)$. It is known from simulations that these are the only stable broken phases ${ }^{4}$. In the gauge $\Omega=1$ the commutator terms in $\vec{B}$ and in the second covariant derivative term do cancel. This property determines the $Y_{k}$ uniquely. For the surviving Abelian expression Stokes theorem is true and a gauge invariant projection of the

${ }^{\mathrm{a}}$ In three dimensions a proof exists ${ }^{5}$. It is based on the confining ground states being due to global magnetic $\mathrm{Z}(\mathrm{N})$ being broken. The unique tension $\sigma_{k}$ is that of the domain wall separating two vacua differing by the phase $\exp \left(i k \frac{2 \pi}{N}\right)$. In $4 \mathrm{~d}$ there is a consensus, based on circumstantial proof. 
line integral results. For the relation of this line integral to the original Wilson loop see ref. ${ }^{3}$.

Both magnetic screening and tension are at high $\mathrm{T}$ dominated by the 3d $S_{M Q C D}$.

\subsection{Electric observables}

Electric observables are the familiar Debye screening mass $m_{D}$ between two heavy quarks, and the mean flux as measured by the spatial 't Hooft loop.

The spatial 't Hooft loop, given by a macroscopic closed $\mathrm{Z}(\mathrm{N})$ magnetic vortex ${ }^{1}$ can be written as a flux loop in the physical Hilbert space:

$$
V_{k}(C)=\exp \left(i \frac{4 \pi}{g} \int d \vec{S} \cdot \operatorname{Tr} \vec{E} Y_{k}\right) .
$$

The surface in the integral is subtended by $C$.

Note the similarity to the first term in eq.(7).

The average of the loop obeys an area law in the deconfined phase, due to the free colour charges in the plasma:

$$
\left.\left\langle V_{k}\right\rangle=\exp \left(-\rho_{k} A\right)\right)
$$

\section{Theoretical results compared to lattice data}

The electric loop can be computed in perturbation theory through integration of the hard modes and Debye modes ${ }^{6}$. This has been done including order $O\left(g^{3}\right)$. Including effects of hard modes to order $g^{4}$ is desirable as will become evident below. The analytic results give Casimir scaling $k(N-k)$ for the contribution of the hard modes.

To get an insight in the analytic results one considers a quasi-particle gas of Debye screened longitudinal gluons with density $n$ per gluon species in the octet (or adjoint for general N). One species should contribute to the tension proportional to its density $\mathrm{n}$. To get the dimension for the tension right one should scale $\mathrm{n}$ by $m_{D}$. In the quasi-particle picture this scaling is simply due to the multiplicity $2 k(N-k)$ of gluons in the adjoint with non-zero $Y_{k}$ charge: $\rho_{k} / m_{D}^{2} \sim k(N-k) n / m_{D}^{3} \sim 1 /(g \sqrt{N})^{3}>>1$, the weak coupling plasma condition.

Below are shown the data for various groups, with Casimir scaling divided out. Remarkably a universal curve results dow to near $T_{c}$ ! Doing the same for the theoretical result gives the curve. Including the hard corrections to $g^{4}$ for the coupling ${ }^{7}$ brings the theoretical curve down. Hence the need for $g^{4}$ for the tension itself. 


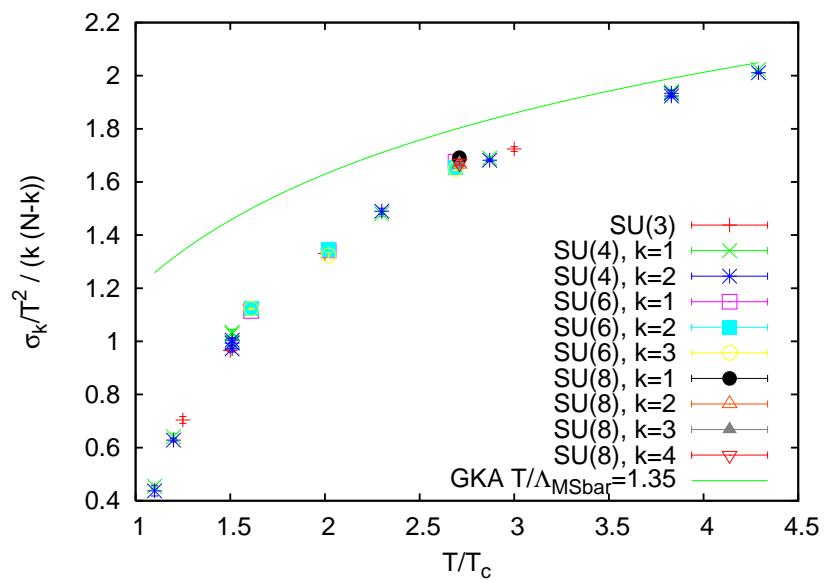

The reduced e-tension for $S U\left(N_{c}\right), N_{c} \leq 8, \mathrm{PdF}$ et al.,hep-lat/0510081

Turning to the magnetic loop, we are faced with its dominant behaviour being due to the non-perturbative magnetic sector. For lack of analytic calculations we proposed ${ }^{8}$ a magnetic quasi-particle model that should explain qualitatively the pressure. As the pressure scales like $N^{2}-1$ to all orders in perturbation theory an adjoint multiplet of dilute monopoles is needed. With an adjoint the same multiplcity argument as for the electric loop is valid (eq.7). So the k-tension of the Wilson loop has again Casimir scaling:

$$
\sigma_{k} \sim k(N-k) n_{M} / m_{M}
$$

The density of a given species is $n_{M}$, the screening of the monopoles is $m_{M}$. We will take it as their size. If that is the case then:

$$
\frac{\sigma_{1}}{m_{M}^{2}} \sim(N-1) \frac{n_{M}}{m_{M}^{3}}
$$

In striking contrast to the weak coupling plasma condition in the electric case in the previous subsection, the left hand side of this relation is for all $\mathrm{N}$ a small number, $\sim 0.05^{10}$. So for finite $\mathrm{N}$ the diluteness $\delta=\frac{n_{M}}{m_{M}^{3}}$ is small. For large $\mathrm{N}$ the l.h.s. is $O(1)$, hence $n_{M}=O(1 / N)$. Below are shown the $3 \mathrm{~d}$ data, which are at most off by two percent.

\section{Effective action for the monopoles}

The raison d'être of the monopole model is that it gives the right group factor for pressure and Wilson loops. At the same time the diluteness is insured 


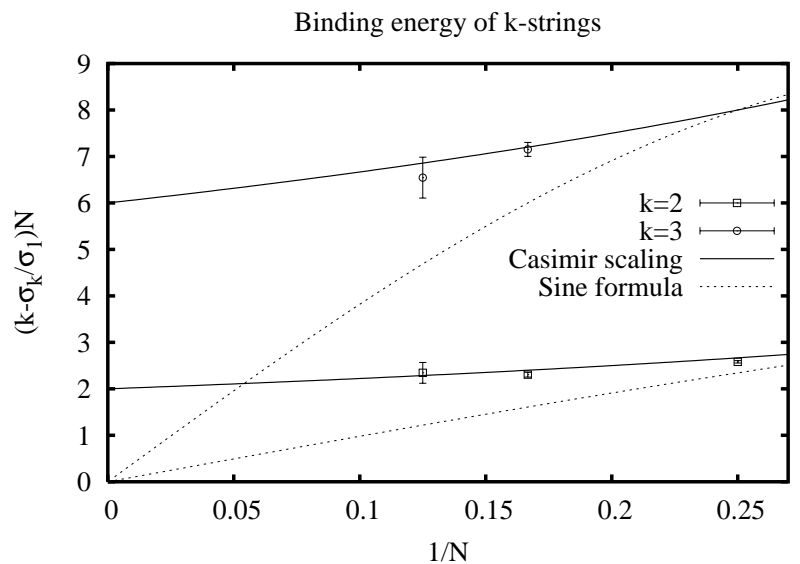

m- flux-tension for $S U\left(N_{c}\right), N_{c} \leq 8$; Meyer, hep-lat/0412021)

by the empirically small ratio $\sigma_{1} / m_{M}^{2}$. This warrants a more methodical approach.

The question is whether one can define an effective action starting from $S_{M Q C D}$. The new action, $S_{M Q P}$, follows by integrating out all scales smaller or equal to the size of the monopole, $m_{M}$. The monopole field $M$ is a traceless hermitean field, transforming under a magnetic global $\mathrm{SU}(\mathrm{N})$ group: $M \rightarrow U M U^{\dagger}$.

The new action should be invariant under this group. This invariance was not there in the magnetostatic action. There are many examples of a symmetry appearing in an effective action and being broken by the higher order terms.

With $S_{M Q P}$ we should be able to compute the k-tensions and the pressure. When computing the pressure we will use the $\overline{M S}$ scheme. The effective action reads in terms of $\mathrm{M}$ scaled by a mass $\sqrt{m_{0}}$ :

$S_{M Q P}=\frac{g_{3}^{6} N^{3}\left(N^{2}-1\right)}{(4 \pi)^{4}}\left(y \log \left(\bar{\mu} / 2 g_{3} N\right)+c\right)+m_{0} \operatorname{Tr}\left[M\left(-\partial^{2} M+m_{0}^{2}\right)(1-\cos M)\right]+\ldots$

where the potential term is that for a dilute Coulomb gas, in analogy with Polyakov ${ }^{1}$.

The first term is the perturbative 4 loop contribution. The coefficient y is known and equals $\frac{43}{12}-\frac{157}{768} \pi^{2}$.

$\bar{\mu}$ is a scale yet to be fixed by perturbative matching. The constant and the mass term $m_{0}=O\left(g_{3}^{2} N\right)$ are unknown. Doing the integration over $M$ 
gives then the pressure:

$$
p_{\bar{M} S}=\frac{g_{3}^{6} N^{3}\left(N^{2}-1\right)}{(4 \pi)^{4}}\left(\left(\frac{43}{12}-\frac{157}{768} \pi^{2}\right) \log \left(\bar{\mu} / 2 g_{3} N\right)+B_{G}+O(\epsilon)\right) .
$$

where $B_{G}=c+f\left(m_{0} / g_{3}^{2} N\right)$.

The short distance behaviour of the cosine potential should be consistent with the first term capturing all of it. A recent lattice determination ${ }^{9}$ gives a result consistent with zero for $B_{G}$. A straightforward computation of the tension $\sigma_{1}$ fixes $m_{0}$.

\section{Conclusions}

We argued that not only electric quasi-particles- the longitudinal gluons but also magnetic quasi-particles (with spin zero as well) explain nicely the flux loop data. These data do not admit dyonic quasi-particles, which would strongly correlate the loops.

What happens to the model when we go down in temperature?

The variation of the ratio $\sigma_{1} / m_{M}^{2}$ from very high $\mathrm{T}$ to $\mathrm{T}=0$ is from about 0.05 to 0.09 . So the gas can still be considered dilute at $\mathrm{T}=0$. If so, $T_{c}$ would the transition where the Bose gas becomes superfluid, and the magnetic symmetry gets spontaneously broken. Vortices in the superfluid become the locus of electric flux strings. Above $T_{c}$ they are absent, so the Polyakov loop develops a VEV above $T_{c}$. One should have experimental signals for this superfluid phase.

I thank Mikko Laine and Harvey Meyer for discussions.

\section{References}

1. G. 't Hooft Nucl. Phys B138, 1 (1978); A. M. Polyakov, Nucl. Phys.B120, 429 (1977).

2. P. de Forcrand, C. P. Korthals Altes, O. Philipsen, to appear in Nucl.Phys.B, hep-ph/0510140 .

3. M. Diakonov, V. Petrov, Phys.Lett.B224, 131 (1989).

4. A. Rajantie, Eger 1997, Strong electroweak matter '97, hep-ph/9709368.

5. C. P. Korthals Altes, H.B. Meyer, in preparation.

6. For a review, see P. Giovannangeli, C.P. Korthals Altes, Nucl.Phys.B721:2549,2005, hep-ph/0412322.

7. Y. Schroeder, hep-lat/03091; M. Laine, Y. Schroeder, JHEP 0503:067,2005.

8. P. Giovannangeli, C. P. Korthals Altes Nucl. Phys.B608, 203 (2001), hepph/0102022; C.P. Korthals Altes, H. B. Meyer, hep-ph/0509018.

9. F. di Renzo, M. Laine, V. Miccio, Y. Schroeder, C. Torrero, hep-ph/0605042.

10. M. Teper, Phys.Rev.D59:014512,1999; hep-lat/980400. 